\title{
A prospective randomized study of colonoscopy using blue laser imaging and white light imaging in detection and differentiation of colonic polyps
}

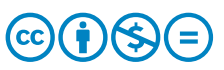

\begin{abstract}
Authors
Institutions

1 Department of Gastroenterology and Hepatology, Changi General Hospital

2 Department of Laboratory Medicine, Changi General Hospital
\end{abstract}

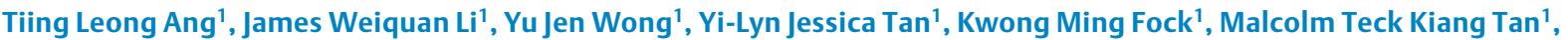
Andrew Boon Eu Kwek¹, Eng Kiong Teo ${ }^{1}$, Daphne Shih-Wen Ang', Lai Mun Wang²

submitted 29.4.2019

accepted after revision 26.6.2019

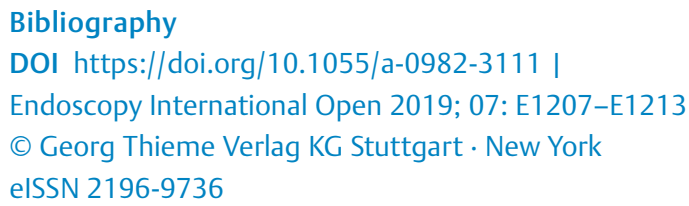

Corresponding author

Tiing Leong Ang, Department of Gastroenterology and Hepatology, Changi General Hospital, 2 Simei Street 3, Singapore 529889

Fax: +6562830402

ang.tiing.leong@singhealth.com.sg

\section{ABSTRACT}

Background and study aims Published data on blue laser imaging (BLI) for detection and differentiation of colonic polyps are limited compared to narrow band imaging (NBI). This study investigated whether BLI can increase the detection rate of colonic polyps and adenomas when com- pared to white light imaging (WLI), and examined use of NICE (NBI International Colorectal Endoscopic) and JNET (Japan NBI Expert Team) classifications with BLI.

Patients and methods Patients aged 50 years and above referred for colonoscopy were randomized to BLI or WLI on withdrawal. Detected polyps were characterized using NICE and JNET classifications under BLI mode and correlated with histology. Primary outcome was adenoma detection rate. Secondary outcomes were utility of NICE and JNET classifications to predict histology using BLI.

Results A total of 182 patients were randomized to BLI (92) or WLI (90). Comparing BLI with WLI, the polyp detection rate was $59.8 \%$ vs $40.0 \%, P=0.008$, and the adenoma detection rate was $46.2 \%$ vs $27.8 \%, P=0.010$. NICE 1 and JNET 1 diagnosed hyperplastic polyps with sensitivity of $87.18 \%$ and specificity of $84.35 \%$. NICE 2 diagnosed low(LGD) or high-grade dysplasia (HGD) with sensitivity of $92.31 \%$ and specificity of $77.45 \%$. JNET 2 A diagnosed LGD with sensitivity of $91.95 \%$, and specificity of $74.53 \%$. Four cases of focal HGD all had JNET 2A morphology.

Conclusion BLI increased adenoma detection rate compared to WLI. NICE and JNET classifications can be applied when using BLI for endoscopic diagnosis of HP and LGD but histological confirmation remains crucial.

\section{Clinical.Trials.gov}

NCT03421600

TRIAL REGISTRATION: Single-Center, randomized, prospective trial NCT03421600 at clinicaltrials.gov

\section{Introduction}

Colorectal cancer (CRC) develops through an adenoma-carcinoma sequence and detection of precancerous colonic adenomas by colonoscopy, and subsequent endoscopic resection, will prevent disease progression, and can be a curative procedure for intramucosal adenocarcinoma [1 - 3].

In contrast to white light imaging (WLI), imaging of the colonic mucosa using a narrow bandwidth enhances visualization of mucosal blood vessels and mucosal pit patterns. Using this principle, narrow band imaging (NBI) (Olympus Corporation, Tokyo, Japan) which is based on use of an optical filter, has been extensively investigated for detection and diagnosis of coIonic polyps. In the context of adenoma detection, results are conflicting. A meta-analysis of randomized studies examining utility of the first-generation NBI system when compared to high-definition WLI showed no difference in detection rates; it was only superior when compared to non-high definition WLI 
[4]. A randomized controlled study utilizing the second-generation NBI system, which has brighter illumination, reported that NBI improved polyp and adenoma detection rates compared to WLI [5]. Conversely, it has been clearly shown that NBI was useful in predicting polyp histology. The NICE (NBI International Colorectal Endoscopic) classification can be applied without magnification whereas the JNET (Japan NBI Expert Team) classification required optical magnification to predict polyp histology $[6,7]$.

Blue laser imaging (BLI) (Lasereo System, Fujifilm Corporation, Tokyo, Japan) is another form of narrow-band imaging (NBI). Instead of using an optical filter for white light to produce narrow bandwidths, the BLI system has a unique feature of illumination using two lasers and a white light phosphor to accomplish visual enhancement of surface vessels and structures. A laser with a wavelength of $450 \mathrm{~nm}$ stimulates the phosphor to irradiate a white-color illumination. The other laser, with a wavelength of $410 \mathrm{~nm}$, is used to enhance blood vessels at shallow depth in the mucosa [8]. Early data have shown its usefulness in predicting histology of mucosal lesions [9, 10]. When this study was first conceived, there were no published data on the role of BLI in polyp detection. Since then, further limited data have been published [11,12]. The NICE and JNET classification systems for polyp diagnosis were developed using NBI. Published data on BLI for detection and differentiation of colonic polyps are limited compared to NBI.

This study aimed to determine whether BLI can increase detection of colonic adenomas when compared to WLI. It also examined use of NICE and JNET classification systems with the BLI system to predict histology. For screening, the BLI bright mode was used and for magnified observation, the BLI mode was used.

\section{Patients and methods}

\section{Study design and setting}

This was a prospective, randomized study comparing BLI with WLI. It was conducted from July 2017 to March 2019 at the Department of Gastroenterology and Hepatology, Changi General Hospital, which is a regional teaching hospital serving the eastern part of Singapore. All patients provided written informed consent for study participation. The study protocol was approved by the institutional review board (CIRB 2016/3054) and registered with Clinicaltrials.gov (NCT03421600).

\section{Patients}

Patients were included if they were aged 50 years or above and referred for colonoscopy for diagnostic evaluation of colonic symptoms, surveillance of colorectal polyps, or colorectal cancer screening. Patients were excluded it they had acute lower gastrointestinal bleeding, familial colorectal cancer syndrome including familial adenomatous polyposis and hereditary nonpolyposis colorectal cancer syndrome, known inflammatory bowel disease, bloody diarrhea, previous colonic resection, previous extensive abdominal or pelvic surgery where colonoscopy may be considered difficult, were considered unsafe for biopsies or polypectomy due to bleeding tendency, or in situations in which complete colonoscopy could not be completed or performed or a patient had severe comorbid illnesses (ASA 3 and above).

\section{Randomization}

Patients were randomized in a 1:1 ratio in blocks of 10 to undergo either BLI or WLI colonoscopy. Randomization was carried out by computer-generated random sequences. Individual random sequence was placed in an opaque envelope and kept by an independent research assistant who was not involved in this study. Once informed consent was obtained, and upon reaching the cecum, the envelope was opened and the assigned imaging technique (BLI or WLI) was disclosed to the endoscopist. The gastrointestinal pathologist reporting on the histology was blinded to the polyp endoscopic appearance based on NICE and JNET classifications.

\section{Technique of colonoscopy and imaging}

Patients received $4 \mathrm{~L}$ of polyethylene glycol in a split dose for bowel cleansing before colonoscopy. An endoscopy system with BLI and WLI functions and optical magnification capability was used (LASEREO, Fujifilm, Tokyo, Japan). Colonoscopy was performed under conscious sedation with intravenous midazolam and/or fentanyl. In the BLI group, insertion to cecum was performed under WLI and once the cecum was reached, the BLI bright mode was switched on during endoscope withdrawal for complete colonic examination. In the WLI group, WLI was used during both insertion and withdrawal. Bowel preparation of the whole colon was graded according to the Boston Bowel Preparation Scale [13].

Size and location of all colonic polyps were recorded contemporaneously. Size of colonic lesions was measured against the span of an opened biopsy forceps. Regardless of the assigned group, once a polyp was detected during withdrawal, prior to removal, the surface structure of each polyp detected was first assessed without optical magnification under BLI bright mode using NICE classification. Thereafter optical magnification was applied and the polyp was classified using JNET classification using BLI mode. Images were captured electronically. All lesions were resected or biopsied and sent for histological examination. All procedures were performed by experienced endoscopists. Prior to the start of the study, NICE and JNET classifications were formally reviewed with all participating endoscopists to ensure familiarity with these classifications for polyp assessment.

\section{Definitions}

Complete colonoscopy was defined as successful cecal intubation. Histological interpretation of all polyps followed the World Health Organization system [14]. Advanced adenoma was defined as adenoma $\geq 10 \mathrm{~mm}$ in diameter, villous histology, high-grade dysplasia (HGD), or intramucosal carcinoma [5]. Adenoma and polyp detection rates were defined as the proportion of patients with at least one adenoma and one polyp respectively. 


\section{Statistics}

The initial sample size estimation was based on the assumption that BLI was superior to WLI for adenoma detection. We estimated the overall prevalence of colorectal adenoma in the WLI colonoscopy group to be $25 \%$. To show a clinically important improvement of adenoma detection by BLI, we assumed that BLI should increase the adenoma detection rate by $15 \%$. With a statistical power of $80 \%$ and a two-sided significance level of 0.05 , 152 patients would be needed in each study arm, such that the total study population was 304 patients. We conducted an interim blinded analysis at study midpoint to assess the trend and to guide us on further conduct of the study in terms of study continuation or early termination. With group sequential analysis, the Pocock boundary gave a $P$ value threshold for each interim analysis which guided the decision on whether to stop the trial. If a single mid-point interim analysis was performed the nominal significance level corresponding to an overall significance level of 0.05 was 0.0294 [15].

Colonic polyp and adenoma detection rates of the BLI and WLI groups were compared using chi-square test. Statistical significance was taken as two-sided $P<0.05$. Using histology as gold standard, sensitivity, specificity, positive and negative predictive values, and accuracy of NICE and JNET classifications were calculated. Statistical analysis was performed using SPSS software (version 20.0; SPSS, Chicago, IL) and MedCalc statistical software (www.medcalc.org).

\section{Results}

\section{Patient characteristics}

During the study period from July 2017 to March 2019, 184 patients were screened. Two were excluded as they did not meet inclusion criteria and 182 patients were randomized to either

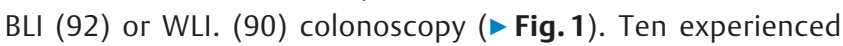
endoscopists performed the study examinations. Mean age of patients was 62.9 years $( \pm 8.5)$ and $56.6 \%$ were men. There was no significant difference in clinical characteristics between the two groups. There was no significant difference in quality of bowel preparation and withdrawal time between the two groups ( $>$ Table 1 ). No complications occurred during colonoscopy.

A total of 194 polyps (sessile or flat: 175; pedunculated: 19; mean size $4 \mathrm{~mm}$ [range: 1 to $20 \mathrm{~mm}$ ] were detected in 91 patients. Polyp histology was hyperplastic in 78, inflammatory pseudo-polyp in one, sessile serrated polyp or adenoma in 22 , adenoma with low-grade dysplasia (LGD) in 88 , and high-grade dysplasia (HGD) in four. One resected polyp could not be retrieved. One patient had advanced rectal adenocarcinoma.

\section{Adenoma detection rate and endoscopic- histological correlation}

Comparing $\mathrm{BLI}$ with $\mathrm{WLI}$, the adenoma detection rate was $46.2 \%$ vs $27.8 \%, P=0.010$. Comparing BLI with WLI, the polyp detection rate was $59.8 \%$ vs $40.0 \%, P=0.008$. NICE 1 and JNET 1 morphology both diagnosed hyperplastic polyps with sensitivity of $87.18 \%$ and specificity of $84.35 \%$. NICE 2 morphology

\section{4 patients randomized}

2 patients excluded

- withdrawal of consent (1)

- age less than 50 years (1)

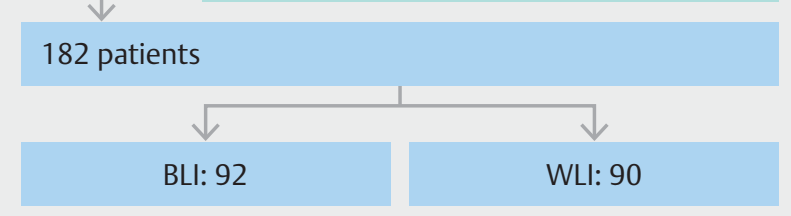

Fig. 1 Trial profile.

- Table 1 Patient characteristics.

\begin{tabular}{|l|c|c|c|}
\hline & BLI (n=92) & WLI (n=90) & P \\
\hline Mean age in years (SD) & $62.5(7.9)$ & $63.2(8.9)$ & 0.609 \\
\hline Male (\%) & $56(60.9 \%)$ & $47(52.2 \%)$ & 0.239 \\
\hline Indications (\%): & & & 0.986 \\
\hline - Cancer screening & $31(33.7 \%)$ & $31(34.4 \%)$ & \\
\hline - Bowel symptoms & $48(52.2 \%)$ & $47(52.2 \%)$ & \\
\hline - Polyp surveillance & $13(14.1 \%)$ & $12(13.3 \%)$ & \\
\hline Complete colonoscopy (\%) & $92(100 \%)$ & $90(100 \%)$ & \\
\hline $\begin{array}{l}\text { Total Boston Bowel Prepa- } \\
\text { ration Score (\%) }\end{array}$ & & & 0.683 \\
\hline - 5 & 0 & $1(1.1 \%)$ & \\
\hline - 6 & $62(67.4 \%)$ & $58(64.4 \%)$ & \\
\hline - 7 & $3(3.3 \%)$ & $1(1.1 \%)$ & \\
\hline - 8 & $5(5.4 \%)$ & $6(6.7 \%)$ & \\
\hline - 9 & $22(23.9 \%)$ & $24(26.7 \%)$ & \\
\hline $\begin{array}{l}\text { Minimum withdrawal time } \\
\text { of } 6 \text { minutes }{ }^{1} \text { (\%) }\end{array}$ & $92(100 \%)$ & $90(100 \%)$ & \\
\hline $\begin{array}{l}\text { BLI, blue laser imaging ; WLI, white-light imaging. } \\
\text { for lesion characterization would lengthen calculation of withdrawal time. }\end{array}$ & \\
\hline
\end{tabular}

diagnosed LGD or HGD with sensitivity of $92.31 \%$ and specificity of $77.45 \%$. JNET 2 A diagnosed LGD with sensitivity of $91.95 \%$, and specificity of $74.53 \%$. The four cases of focal HGD all had JNET 2A morphology ( Fig. 2, > Fig. 3, > Fig. 4, > Fig.5, > Fig. 6, - Fig. 7, > Fig. 8 and $>$ Table 2).

\section{Discussion}

It is crucial to maximize adenoma detection rates (ADRs) to improve long term outcomes in patients with colorectal neoplasia. The use of image enhanced endoscopy (IEE), the focus of the current study, is just one aspect of the overall strategy. The cornerstone for achieving this is good-quality endoscopy 

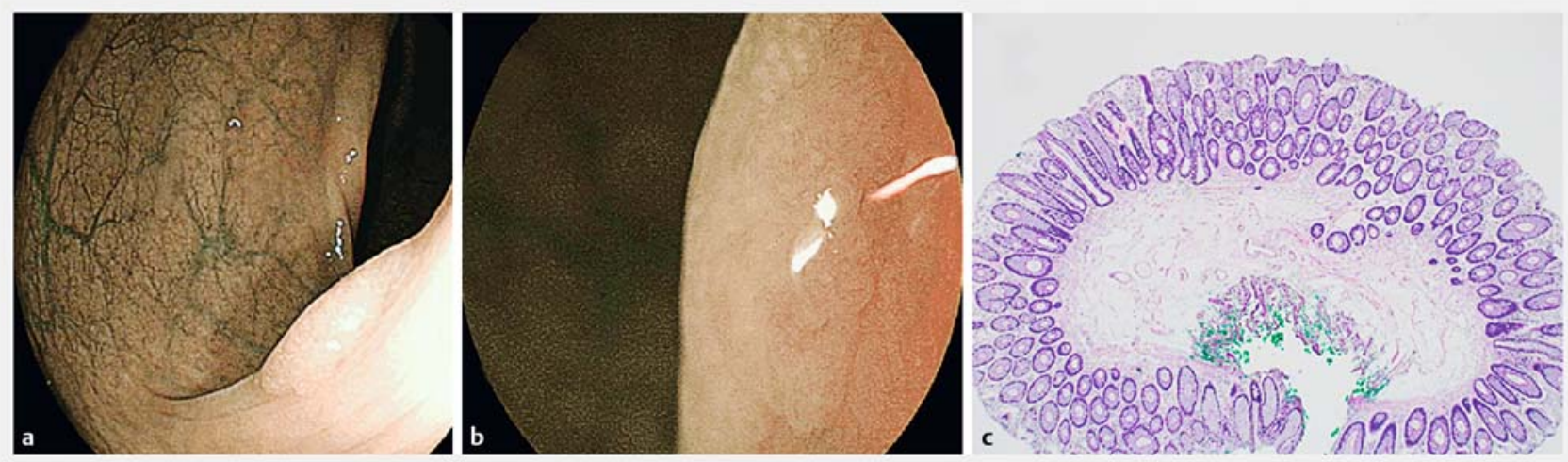

- Fig. 2 a Rectal polyp with NICE 1 and b JNET 1 endoscopic appearance. c Hematoxylin \& eosin-stained section showed features of a hyperplastic polyp with no dysplasia ( $40 \times$ magnification).
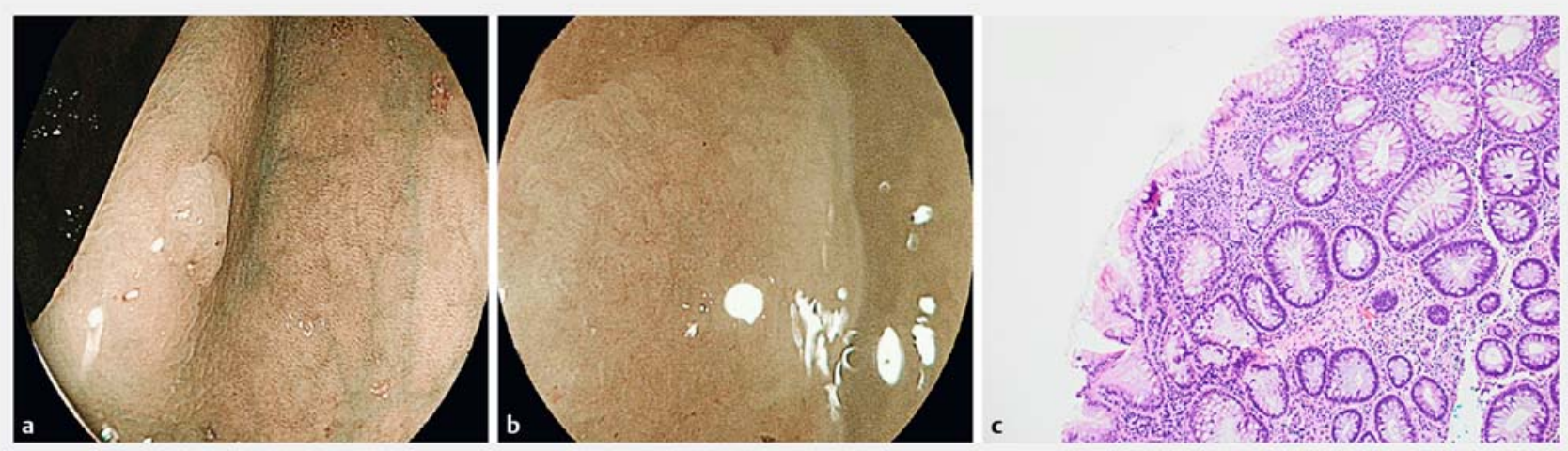

- Fig. 3 a Ascending colon polyp with NICE 1 and b JNET 1 endoscopic appearance. c Hematoxylin \& eosin-stained section showed colonic mucosa with dilation and horizontalization of the basal crypt glands and focal serration, consistent with a sessile serrated adenoma, without conventional cytological dysplasia. (100 × magnification).
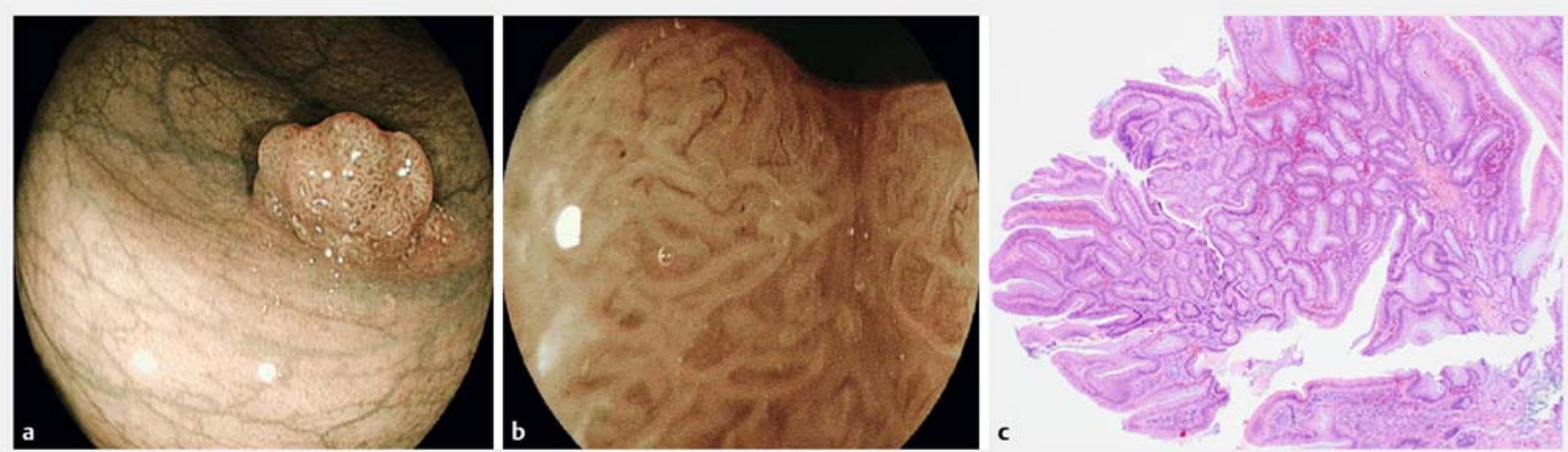

- Fig. 4 a Rectal polyp with NICE 2 and b JNET 2A endoscopic appearance. c Hematoxylin \& eosin-stained section showed features of a tubulovillous adenoma with low-grade dysplasia ( $40 \times$ magnification).

performed by individual endoscopists, with surrogate markers being good bowel preparation, slow withdrawal time, and the individual endoscopist's personal ADR [16]. Other strategies that have been investigated to further enhance ADR include use of IEE as in this study, use of add-on devices, use of fullspectrum endoscopy system (FUSE) as well artificial intelligence $[17,18]$. 

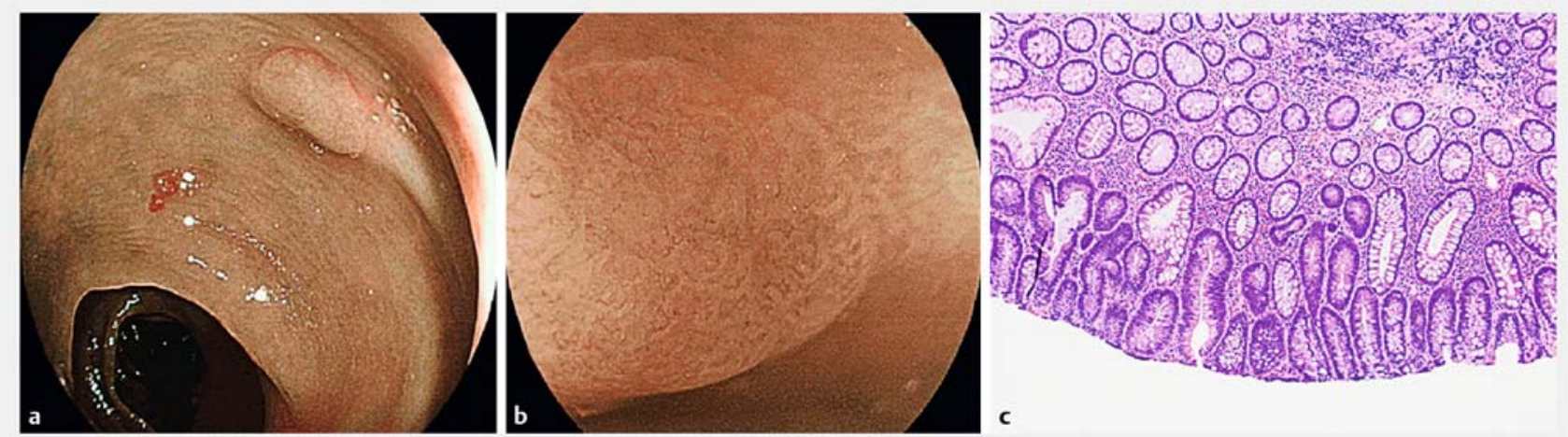

- Fig. 5 a Sigmoid polyp with NICE 1 and b JNET 1 endoscopic appearance. $\mathbf{c}$ Hematoxylin \& eosin-stained section showed features of a tubular adenoma with low-grade dysplasia (100 × magnification).
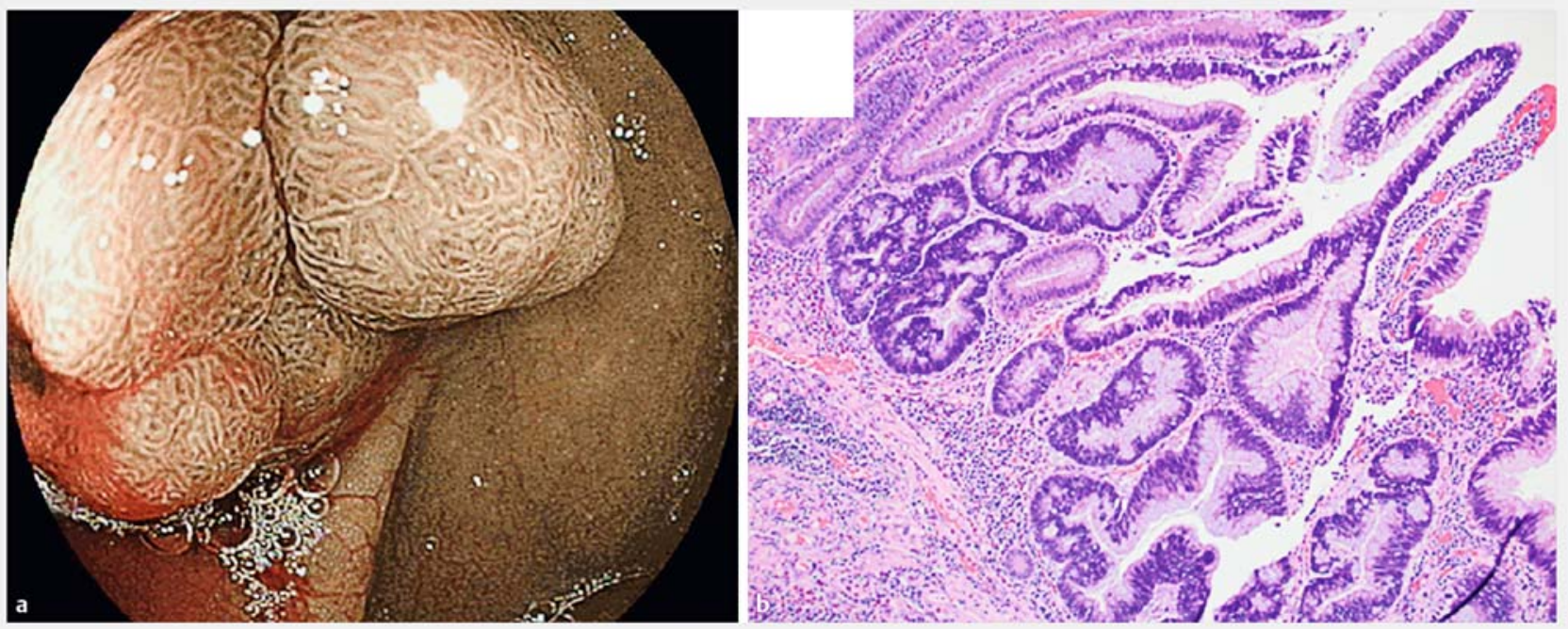

- Fig. 6 a Cecal polyp with NICE 2 endoscopic appearance. b Hematoxylin \& eosin-stained section showed focal high-grade dysplasia within a tubulovillous adenoma with predominantly low-grade dysplasia $(100 \times$ magnification $)$.
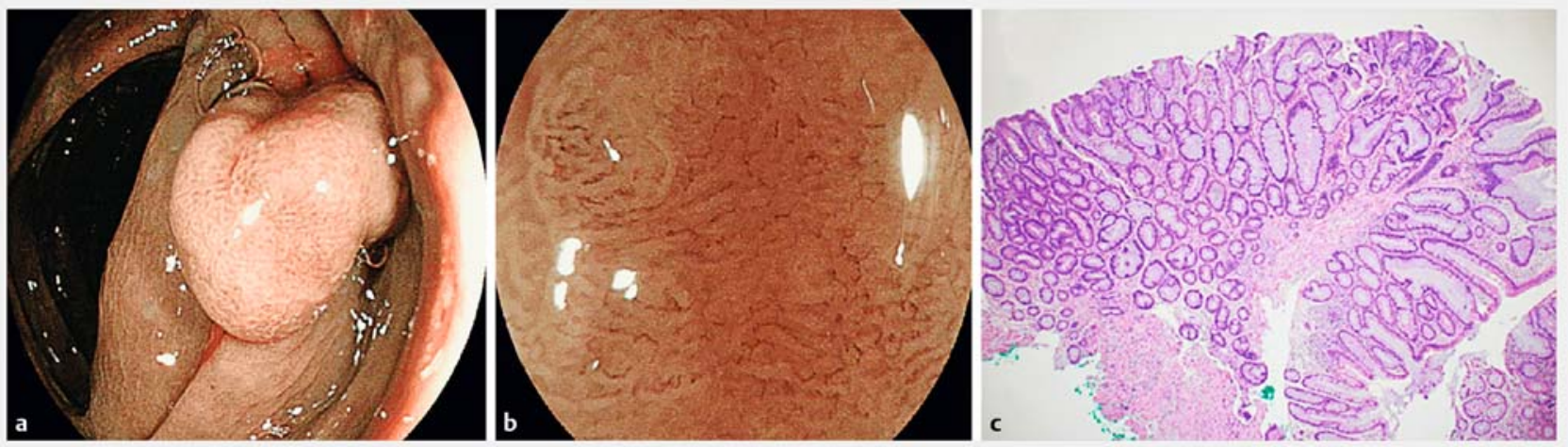

- Fig. 7 a Transverse colon polyp with NICE 2 and b JNET 2A endoscopic appearance. c Hematoxylin \& eosin-stained section showed features of a sessile serrated adenoma with low-grade dysplasia ( $40 \times$ magnification). 

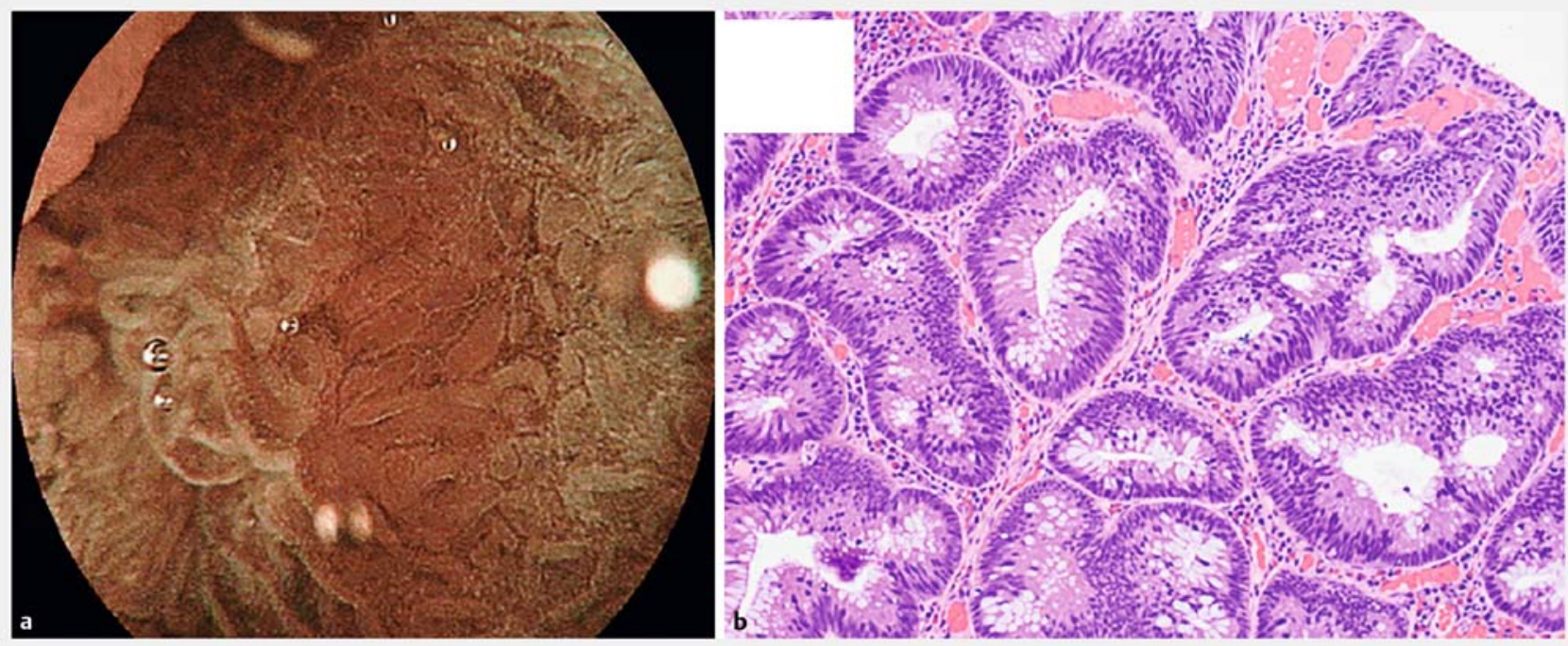

- Fig. 8 a Sigmoid polyp with JNET 2A endoscopic appearance. b Hematoxylin \& eosin-stained section showed focal high-grade dysplasia within a tubular adenoma exhibiting predominantly low-grade dysplasia (200 × magnification).

- Table 2 Performance characteristics for endoscopic prediction of histology.

\begin{tabular}{|c|c|c|c|c|c|}
\hline & $\begin{array}{l}\text { Sensitivity } \\
(95 \% \mathrm{Cl})\end{array}$ & $\begin{array}{l}\text { Specificity } \\
(95 \% \mathrm{Cl})\end{array}$ & $\begin{array}{l}\text { Positive predictive } \\
\text { value }(95 \% \mathrm{Cl})\end{array}$ & $\begin{array}{l}\text { Negative predictive } \\
\text { value }(95 \% \mathrm{Cl})\end{array}$ & $\begin{array}{l}\text { Accuracy } \\
(95 \% \mathrm{Cl})\end{array}$ \\
\hline $\begin{array}{l}\text { Hyperplastic polyp } \\
\text { (NICE } 1 / \text { JNET 1) }\end{array}$ & $\begin{array}{l}87.18 \% \\
(77.68-93.68)\end{array}$ & $\begin{array}{l}84.35 \% \\
(76.40-90.45)\end{array}$ & $\begin{array}{l}79.07 \% \\
(71.02-85.34)\end{array}$ & $\begin{array}{l}90.65 \% \\
(84.40-94.56)\end{array}$ & $\begin{array}{l}85.49 \% \\
(79.72-90.14)\end{array}$ \\
\hline $\begin{array}{l}\text { Sessile serrated polyp } \\
\text { (NICE } 1 / \text { JNET 1) }\end{array}$ & $\begin{array}{l}46.43 \% \\
(27.51-66.13 \%)\end{array}$ & $\begin{array}{l}55.76 \% \\
(47.83-63.47)\end{array}$ & $\begin{array}{l}15.12 \% \\
(10.35-21.55)\end{array}$ & $\begin{array}{l}85.98 \% \\
(80.89-89.88)\end{array}$ & $\begin{array}{l}54.40 \% \\
(47.10-61.57)\end{array}$ \\
\hline $\begin{array}{l}\text { Adenoma with low- or high- } \\
\text { grade dysplasia (NICE 2) }\end{array}$ & $\begin{array}{l}92.31 \% \\
(84.79-96.85)\end{array}$ & $\begin{array}{l}77.45 \% \\
(68.11-85.14)\end{array}$ & $\begin{array}{l}78.50 \% \\
(71.72-84.02)\end{array}$ & $\begin{array}{l}91.86 \% \\
(84.51-95.86)\end{array}$ & $\begin{array}{l}84.46 \% \\
(78.56-89.26)\end{array}$ \\
\hline $\begin{array}{l}\text { Adenoma with low-rade } \\
\text { dysplasia (JNET 2A) }\end{array}$ & $\begin{array}{l}91.95 \% \\
(84.12-96.70)\end{array}$ & $\begin{array}{l}74.53 \% \\
(65.14-82.49)\end{array}$ & $\begin{array}{l}74.77 \% \\
(68.02-80.50)\end{array}$ & $\begin{array}{l}91.86 \%( \\
84.61-95.86)\end{array}$ & $\begin{array}{l}82.38 \% \\
(76.26-87.48)\end{array}$ \\
\hline
\end{tabular}

NICE, NBI International Colorectal Endoscopic; JNET Japan NBI Expert Team.

Our study focused on BLI, a type of IEE that utilizes a narrow bandwidth light source to accentuate mucosal surface contrast. Our study demonstrated that BLI could increase ADR compared to WLI. In addition, it validated use of NICE and JNET classification in the context of BLI for patients with hyperplastic polyps and adenomatous polyps with LGD. Previous publications applied NICE and JNET classifications only in the context of using $\mathrm{NBI}$. It is not surprising that studies using the old generation $\mathrm{NBI}$ systems did not demonstrate any benefit in the context of polyp detection, because dark illumination hampers far-view visualization [4]. To date only one other study has demonstrated that IEE using NBI can increase polyp and ADR [5]. Leung et al reported that when the new-generation NBI system was compared with WLI, it significantly increased polyp and ADR. The newer-generation endoscopy systems with NBI, be it NBI in the study by Leung, or BLI in our study, combine the ability to accentuate mucosa surface details, which is crucial for detailed examination of a lesion, with the benefit of a brighter light source, thus improving visualization of distant lesions.
Even then, having good bowel preparation is especially crucial during colonoscopy with IEE techniques, as suboptimal bowel preparation would interfere with endoscopic visualization more than WLI, due to the darker appearance.

In the study by Leung, both ADR and polyp detection rates were significantly higher in the NBI group compared with the WLI group (adenoma: $48.3 \%$ vs. $34.4 \%, P=0.01$; polyps: $61.1 \%$ vs. $48.3 \%, P=0.02)$. The mean number of polyps detected per patient was also higher in the NBI group (1.49 vs. $1.13, P=$ $0.07)$ [5]. Three other prospective randomized controlled trials utilizing brighter narrow bandwidth technology (1 NBI and 2 BLI) have been published so far $[11,12,19]$. In the other study using NBI, Horimatsu used the Olympus next-generation NBI system with either standard-definition (SD) or wide-angle (WA) colonoscopy and stratified patients into four groups: SD WLI v SD NBI, and WA WLI vs WA NBI. The primary endpoint of the study was mean number of polyps detected per patient. The mean number of polyps detected per patient was significantly higher in the NBI group than in the WLI group (2.01 vs. 
1.56; $P=0.032$ ) [19]. Ikematsu randomized patients to WLI or $\mathrm{BLI}$ with mean number of adenomas per patient as the primary outcome. This was significantly higher in the BLI group (1.27 vs. $1.101, P=0.008)$. There was no difference in ADR between the BLI and WLI groups (54.8\% vs. 52;7\%, $P=0.521)$ [11]. Shimoda randomized patients to tandem colonoscopy with BLI followed by WLI (BLI-WLI group) or WLI followed by WLI (WLI-WLI group). The main outcome measure was the adenoma miss rate. The miss rate in the BLI-WLI group was (1.6\%), which was significantly less than that in the WLI-WLI group $(10.0 \%, P=$ 0.001 ) [12]. Our study differed from the other BLI studies by focusing on $A D R$, rather than mean number of adenomas per patient [11] or adenoma miss rates [12].

The NICE and JNET classification systems were developed using NBI. There had been no prior validation of these systems with histopathological correlation using BLI. Our study formally examined application of NICE and JNET using BLI, which has not been previously published. Our study showed that similar to $\mathrm{NBI}$, these classification systems could be applied using BLI to predict polyp histology. Nonetheless, there are discrepancies between endoscopic and histological diagnoses, thus histological correlation is still important. The classification systems could not reliably diagnose sessile serrated polyps or adenomas. Images of the four cases with HGD were reviewed and confirmed to be truly JNET 2A in appearance and not JNET 2B. On histology, the HGD component of these cases was focal (less than $10 \%$ of the entire adenoma), located towards the basal aspect without extension to the surface of the mucosa and often lacked the well-established microvasculature of an advanced adenoma. Hence, these early HGD foci are not visible endoscopically.

In terms of study strength, this was an investigator-initiated, randomized controlled study performed by experienced endoscopists who regularly used NBI in routine clinical practice. Thus it was not difficult to apply BLI. There was formal pretrial training to ensure that all endoscopists were familiar with the endoscopy system and use of the NICE and JNET classification systems. We acknowledge our study limitations. This was a single-center study with relatively small sample size. However, this was because we terminated our study earlier as the actual effect size was larger than initially calculated. In addition, there were no adenomas with JNET2B morhology in our cohort of patients. Withdrawal time does impact on detection rate, and in our study, we looked at withdrawal time from the perspective of a threshold minimum of 6 minutes, rather than the difference between groups of actual mean withdrawal time.

\section{Conclusion}

To conclude, BLI increased colonic ADR. NICE and JNET classifications could be used to predict hyperplastic or adenomatous polyps.

\section{Competing interests}

\section{References}

[1] Winawer S], Zauber AG, Ho MN et al. Prevention of colorectal cancer by colonoscopic polypectomy. The National Polyp Study Workgroup. N Engl J Med 1993; 329: 1977 -1981

[2] Zauber AG, Winawer S], O'Brien M] et al. Colonoscopic polypectomy and long-term prevention of colorectal-cancer deaths. N Engl J Med 2012; 366: 687-696

[3] Fisher DA, Shergill AK, Early DS et al. Role of endoscopy in the staging and management of colorectal cancer. Gastrointest Endosc 2013; 78: $8-12$

[4] Nagorni A, Bjelakovic G, Petrovic B. Narrow band imaging versus conventional white light colonoscopy for the detection of colorectal polyps. Cochrane Database Syst Rev 2012; 1: CD008361

[5] Leung WK, Lo OS, Liu KS et al. Detection of colorectal adenoma by narrow band imaging (HQ190) vs. high-definition white light colonoscopy: a randomized controlled trial. Am J Gastroenterol 2014; 109: $855-863$

[6] Sano Y, Tanaka S, Kudo SE et al. Narrow-band imaging (NBI) magnifying endoscopic classification of colorectal tumors proposed by the Japan NBI Expert Team. Dig Endosc 2016; 28: 526- 533

[7] Sumimoto K, Tanaka S, Shigita K et al. Clinical impact and characteristics of the narrow-band imaging magnifying endoscopic classification of colorectal tumors proposed by the Japan NBI Expert Team. Gastrointest Endosc 2017; 85: 816-821

[8] Yoshida N, Yagi N, Inada Y et al. Ability of a novel blue laser imaging system for the diagnosis of colorectal polyps. Dig Endosc 2014; 26: $250-258$

[9] Yoshida N, Hisabe T, Inada Y et al. The ability of a novel blue laser imaging system for the diagnosis of invasion depth of colorectal neoplasms. J Gastroenterol 2014; 49: $73-80$

[10] Yoshida N, Hisabe T, Hirose R et al. Improvement in the visibility of colorectal polyps by using blue laser imaging (with video). Gastrointest Endosc 2015; 82: $542-549$

[11] Ikematsu H, Sakamoto T, Togashi K et al. Detectability of colorectal neoplastic lesions using a novel endoscopic system with blue laser imaging: a multicenter randomized controlled trial. Gastrointest Endosc 2017; 86: 386-394

[12] Shimoda R, Sakata Y, Fujise T et al. The adenoma miss rate of blue-laser imaging vs. white-light imaging during colonoscopy: a randomized tandem trial. Endoscopy 2017; 49: 186-190

[13] Lai EJ, Calderwood AH, Doros G et al. The Boston bowel preparation scale: a valid and reliable instrument for colonoscopy-oriented research. Gastrointest Endosc 2009; 69: 620-625

[14] Hamilton SR, Aaltonen LA (eds) World Health Organization Classification of Tumours. Pathology and Genetics of Tumours of the Digestive System2nd edn. Lyon, France: IARC Press; 2000

[15] Pocock SJ. Size of cancer clinical trials and stopping rules. Br J Cancer 1978; 38: 757-766

[16] Rex DK, Schoenfeld PS, Cohen J et al. Quality indicators for colonoscopy. Gastrointest Endosc 2015; 81: $31-53$

[17] Gkolfakis P, Tziatzios G, Facciorusso A et al. Meta-analysis indicates that add-on devices and new endoscopes reduce colonoscopy adenoma miss rate. Eur J Gastroenterol Hepatol 2018; 30: 1482 - 1490

[18] Kudo SE, Mori Y, Misawa M et al. Artificial intelligence and colonoscopy: current status and future perspectives. Dig Endosc 2019; 31: $363-371$

[19] Horimatsu T, Sano Y, Tanaka S et al. Next-generation narrow band imaging system for colonic polyp detection: a prospective multicenter randomized trial. Int J Colorectal Dis 2015; 30: 947 - 954 